\title{
ESTUDIOS
}

\section{QUELQUES POINTS DE DEPART POUR DES STANDARDS EUROPÉENS CONCERNANT LA QUALIFICATION DES CONSEILLERS PROFESSIONNELS}

\section{BASIC ASSUMPTIONS ON EUROPEAN GUIDANCE COUNSELLOR QUALIFICATION STANDARDS}

\author{
Prof. Dr. B-J. Ertelt*
}

\section{RÉSUMÉ}

De nombreuses organisations europénnes soulignent ces temps-ci l'importance des conseillers et posent la question de leur professionalisation. Leur travail sert en effet aux gouvernements, aux entreprises, aux organismes de formation, autant qu'aux personnes demandeuses de conseils. Dans ce contexte, il est urgent d'harmoniser les formations des conseillers européens afin qu'ils proposent un service efficace et adapté. L'apprentissage tout au long de la vie concerne aussi les conseillers dont le travail devrait etre guidé par des principes éthiques et peut-être aussi par une instance de contrôle, ce qui néanmoins pose des problèmes en Allemagne, par exemple.

Le présent article adresse un état des lieux de la question, insiste sur les points de convergence et de divergence entre les différents standards européens pour la qualification des conseillers. Enfin, il présente des suggestions pour affermir et harmoniser ces standards, notamment en ce qui concerne la formation diplomante des conseillers, les standards éthiques et l'instance de contrôle professionnelle.

Mots clé: Professionalisation, qualification des conseillers, formation post-universitaire, standards éthiques, organisation professionnelle, gestion, qualité

\begin{abstract}
Several European organisations acknowledge the importance of the work of guidance counsellors and raise the issue of their training and professional development. It is urgent to standardise the training of
\end{abstract}

* El Dr. Bernd-Joachim ERTELT es profesor de pedagogía profesional y económica en la Escuela Superior Federal para la Administración del Trabajo, en Mannheim, Alemania. Su docencia e investigación se centra sobre todo en las comparaciones internacionales de la formación de los orientadores en la dimensión profesional, y en la gestión de la información en el campo de la orientación profesional. Dirección de contacto: FH Bund Fachbereich Arbeitsverwaltung; Postfach 101061; D-68010 Mannheim. TEL:0049-(0)621/4209-140 FAX: 0049-(0)621/4209-197 Correo-e: bernd-joachim.ertelt@arbeitsamt.de 
European guidance counsellors so that they can adequately meet the demands of their clients, who in spite of the differences among their countries, face similar problems regarding education and training.

This article addresses the state of the art, insisting on the points of convergence and divergence among the different sets of European qualification standards for guidance counsellors, and presents the contents of some training modules to deliver these standards. It concludes with some suggestions to consolidate and harmonise these standards, especially in relation to post-graduate training, and ethical standards.

Key words: professional development, counsellor qualification standards, post-graduate training, ethical standards, management, quality

\section{RESUMEN EN CASTELLANO}

Diversos organismos europeos han reconocido la importancia del trabajo de los orientadores y plantean la cuestión de su profesionalización. En este artículo se defiende la necesidad de reflexionar sobre y proponer unos estándares de formación para los orientadores en Europa. Se justifica en primer lugar la importancia de la orientación profesional en el seno de la Unión Europea, patente en diversos documentos, declaraciones, informes, y/o programas; tales como la «cumbre de empleo» del Consejo de Europa para el Empleo, el Programa LEONARDO DA VINCI II, el informe de la ODCE sobre los jóvenes y la orientación profesional, o el Memorando de la Comisión Europea sobre el aprendizaje a lo largo de toda la vida, entre otros. Asimismo se argumenta que en la era de la globalización y la «interdependencia internacional» los problemas y retos educativos y profesionales a los que se enfrentan los ciudadanos europeos son muy similares, a pesar de las diferencias entre los distintos países.

En segundo lugar se señalan los puntos de convergencia y divergencia entre los diferentes estándares europeos de formación de orientadores, y se presenta una propuesta concreta, con determinados contenidos estructurados en MODULOS formativos para capacitar a los orientadores en las tareas que mayoritariamente deben realizar para desempeñar su trabajo en un contexto trans-nacional.

Por último, después de comparar a grandes rasgos la modalidad de formación de los orientadores entre diversos países europeos, se reflexiona sobre la adecuación o no de adoptar unos estándares europeos en la formación de sus profesionales de la orientación, su utilidad y las condiciones necesarias para que puedan realmente adoptarse, ofreciendo a la vez unas sugerencias para consolidarlos, básicamente en la formación continua o de postgrado.

Palabras clave: Profesionalización, estándares de formación de orientadores, formación de post-grado, normas éticas, organización profesional, gestión, calidad.

\section{Importance de l'orientation professionnelle et de la formation des conseillers}

Aussi similaires que soient les questions et les problèmes, d'autant plus diverses sont les réponses selon les différents pays. La cause de ces différences entre les systèmes d'orientation et d'information ainsi que de placement tient certainement aux structures socio-économiques et juridiques-institutionnelles. Celles-ci concernent l'accès, le degré d'approvisionnement, l'orientation vers les groupes cibles, le volume des prestations de service, la qualification du personnel et la méthodologie.

A l'égard de la fonction de l'orientation professionnelle dans le monde du travail en évolution, la Commission de l'UE et d'autres organisations internationales comme l'OCDE ont fait des déclarations de plus en plus précises. 
Lors du «sommet d'emploi» extraordinaire European Council on Employment (Luxembourg, 20-21 novembre 1997), l'orientation professionnelle recevait une nouvelle dimension, elle devenait un outil de la politique de l'emploi.

Ainsi dans le programme de l'UE LEONARDO DA VINCI II (2000-2006), l'orientation professionnelle a plus d'importance qu'avant.

L'étude de l'OCDE «Mapping the Future - Young People and Career Guidance» (1996) offre un troisième point de référence pour la discussion des standards européens concernant la qualification des conseillers. On peut faire la liste des objectifs et des avantages pour une orientation professionnelle efficace:

Pour les gouvernements et la société

- L'orientation professionnelle est un outil pour l'allocation optimum au sens d'une utilisation plus efficace et plus économique des ressources humaines.

- Chaque société a un intérêt fondamental à l'attribution et au maintien de certains postes professionnels.

Pour les employeurs

- Recrutement de la main-d'œuvre adaptée.

- Développement de l'effectif du personnel.

- Dévision du besoin en personnel.

Pour l'école, les écoles supérieures, les organismes de formation

- Mieux adaptés les programmes d'éducation aux besoins des participants.

- Réduction de la quote-part des «décrocheurs» dans l'éducation et la formation (continue).

Pour l'indivudu

- Meilleure conception du passage de l'école à la formation professionnelle et à l'emploi.

- Meilleure utilisation des offres d'éducation et de formation.

- Améliation du choix autodéterminé d'une profession

- Promotion de décisions efficaces et réalistes dans le processus du choix d'une profession.

Afin d'atteindre de nombreuses recommandations et ces objectifs, l'étude de l'OCDE fait aux acteurs responsables pour le perfectionnement de l'orientation professionnelle:

1. garantir le nombre nécessaire de conseillers

2. assurer une formation hautement qualifiée des conseillers

3. définir des standards solides pour l'orientation professionnelle et favoriser l'identité professionnelle

4. garantir un service d'orientation plus efficace et plus étendu pour les défavorisés

5. accélérer le développement du bon matériel d'orientation par une coopération internationale

6. faire ressortir le rôle de l'orientation professionnelle en public 
7. promouvoir une collaboration étroite de tous services d'orientation correspondants surtout au niveau local

8. le système d'éducation doit rendre capables les individus de prendre suffisamment tôt des décisions professionnelles autonomes, afin d'en décharger l'orientation professionnelle

9. développer des méthodes innovantes en matière d'information professionnelle

10. prendre l'orientation professionnelle plus au sérieux, surtout à l'égard des réactions des demandeurs de conseil.

Une quatrième raison importante pour réfléchir à des standards dans la qualification des conseillers est l'observation que, à l'ère de la mondialisation croissante et de l'interdépendance internationale, les problèmes des citoyens dans les pays européens sont étonnamment similaires: partout se posent des questions lors de la carrière scolaire, du choix d'une profession, lors du passage à la formation professionnelle ou au système d'emploi, lors de la mobilité professionnelle, lors d'une perte d'emploi, lors de l'intégration professionnelle des gens socialement défavorisés, lors de la reprise d'une activité professionnelle par les femmes, lors de l'intégration des minorités éthniques ou des réfugiés, lors de la réduction des obstacles de classe sociale et lors de la lutte contre le chômage des jeunes ou lors du chômage de longue durée.

Dans le memorandum d'octobre 2000 de la Commission de l'UE sur l'appentissage tout au long de la vie il y a notamment six messages sur la nécessité d'une orientation professionnelle:

- Les informations par l'Internet, prises séparément, ne sont pas encore de l'orientation professionnelle. Il faut y ajouter la classification des informations dans la situation individuelle du demandeur de conseil et la vérification de leur justesse et de leur validité.

- Dans les administrations du travail publiques (Public Employment Services, PES) les services d'orientation professionnelle pour les adolescents et les adultes doivent jouer un rôle central avec le soutien de l'aptitude individuelle à l'emploi.

- Dans la société du savoir, de la communication imbriquée et du changement qui y est lié, une orientation professionnelle européenne doit apporter une contribution importante à la citoyenneté active et à l'aptitude à l'emploi.

- L'appentissage tout au long de la vie, la protection de l'aptitude à l'emploi comme buts politiques nationaux nécessitent une mise en œuvre concrète au niveau régional et local. La condition préalable pour l'efficience est l'interconnexion économisante des ressources, entre des services d'orientation et de placement, qui existent dans les organismes de formation et de formation continue ainsi que dans les entreprises.

- Il faut augmenter les services de consultation pour les entreprises (consultation pour les employeurs, direction des ressources humaines, coaching, out-placement).

L'orientation professionnelle doit promouvoir aussi le sens des affaires parmi les employés au sens de «l'entrepreneurship». 


\section{Les différents standards européens pour la qualification des conseillers}

Sur ce fond on va traiter les tendances de l'orientation professionnelle dans trois domaines exemplaires: le point de départ méthodologique, la gestion qualité et la gestion des ressources humaines dans les entreprises.

Par rapport à la méthodologie d'orientation professionnelle, la gestion d'information est sûrement au centre des défis actuels.

Mais une autre tâche de développement importante se pose aussi sur la conception des processus de transition, dont le passage de l'école à la vie active mérite une considération particulière.

Un troisième axe essentiel des tendances méthodologiques concerne l'intégration des tâches de placement dans l'orientation professionnelle. Il ne s'agit pas du tout d'un changement de paradigme vers l'aspect de placement de l'orientation professionnelle, mais de la plus grande prise en considération de l'aspect de réalisation, comme souvent souhaité par des demandeurs de conseil.

Il manque surtout des critères solides généralement reconnus pour une gestion qualité de l'orientation professionnelle, et des standards appropriés pour la qualification et l'activité de conseillers professionnels. Ainsi, le Code social III, entré en vigueur en 1998, avec son règlement relatif aux conditions minimum pour l'activité en tant que conseiller, présente un exemple négatif au sens des grandes exigences traitées au début, à l'égard de l'orientation professionnelle en Europe.

Des efforts semblent d'autant nécessaires de la part des organismes spécialisés comme l'AIOSP et des associations professionnelles nationales dans les pays membres.

En élaborant des programmes de formation, on devrait tout d'abord considérer des champs concrets. Cependant il faut ajouter à la classification scientifique technique, l'examen de la contribution au développement de la personnalité professionnelle et à l'utilité sociale. On ne devrait pas oublier non plus les aspects professionnels d'ordre politique.

Par des études comparatives des profils professionnels dans le domaine d'activité d'orientation professionnelle en Europe (cf. Watts, 1993), on peut constater les suivantes activités principales - toutefois avec un problème de délimitation:

En dehors des profils d'activité, il y a des standards éthiques ou des directives des grandes organisations professionnelles nationales ou internationales qui donnent des indices importants pour la formation qualifiante des conseillers.

Par exemple le Code de déontologie canadien, qui s'appuie sur des modèles des U.S.A., définit les standards de qualification (article 10) comme suit: «Un conseiller devrait avoir une qualification généralement reconnue dans son métier et faire des efforts pour maintenir sa compétence professionnelle par une formation continue permanente» (Ertelt/Schulz, 1997).

Et dans les standards éthiques de l'Association internationale d'orientation scolaire et professionnelle (AIOSP) de 1996 on peut lire, entre autres: «Les membres de l'AIOSP suivent la formation appropriée et poursuivent un processus d'apprentissage dans tous les domaines du savoir et des aptitudes qui sont nécessaires pour être un conseiller compétent d'éducation et d'orientation». 


\section{TABLEAU 1: Champ d'Activité des Conseillers d'Orientation Professionelle en EC (1993)}

1. Gestion d'informations

- Collecte d'informations, traitement et communication en fonction de personnes cibles

- Exploration de nouvelles sources d'information

- Evaluation des supports d'information en fonction de leur performance

- Marketing de l'information

2. Conseil individuel (y compris appréciation des aptitudes et des prestations)

3. Travail avec des groupes

4. Procuration de places de formation et d'emplois, dévelopment du personnel/gestion du personnel de l'entreprise

5. Management

Dans le synopsis les sources nommées permettent de reconnaître les contours des standards européens pour le contenu et le niveau d'une qualification des conseillers. Il s'agit non seulement de la compétence technique et méthodologique, mais aussi de la compétence sociale et de la morale professionnelle. Alors, on ne doit pas tomber dans l'oubli que «l'accord parfait» des personnalités des acteurs dans un entretien d'orientation a une influence considérable sur le succès. Cela pourrait signifier une certaine relativisation de l'importance des qualifications formelles et une plus grande attention aux caractéristiques personnelles comme conditions d'admission à la profession de conseiller.

Néanmoins on va faire des propositions pour certains contenus d'une formation qualifiante européenne des conseillers. Puis nous présenterons des exemples en cours d'études en Europe et enfin nous discuterons des recommandations pour des standards.

\section{Des suggestions pour affermir et harmoniser ces standards}

Les diverses sources sont d'accord sur le fait qu'on ne peut garantir une orientation professionnelle responsable, neutre et efficace, que par une professionnalisation avancée des acteurs d'orientation. L'élément essentiel de cette orientation reste la liberté individuelle des jeunes gens lors des décisions professionnelles.

Les opinions sont divergentes sur la question de savoir si cette professionnalisation et la garantie de standards, absolument nécessaires dans l'orientation professionnelle, demandent un profil professionnel régi par la loi. Il y avait des voix (cf. Watts et al., 1993) mettant en garde contre un «modèle traditionnel» comme l'ordre professionnel valable pour les médecins ou les avocats, par exemple. Mais, en revanche, d'autres considèrent des règles de droit relatives à la qualification, l'admission et l'exercice comme des dispositions de protection indispensables aux clients (p.ex. l'Association allemande d'orientation professionnelle $[\mathrm{dvb}])$. 


\section{TABLEAU 2: STANDARDS EUROPÉENS dans la formation de conseillers d'orientation}

\section{GESTION D'INFORMATION TRANSNATIONALE}

Module 1: Éducation, formation, études, vie et travail, services d'orientation et d'information dans les pays européens

Module 2: Participation à la mise en place des systèmes d'information pour l'orientation transnationale et centrée sur l'Europe

Module 3: Évaluation des médias et des systèmes d'information par capacité, analyse du ratio coût à profit

2. ORIENTATION ET INFORMATION

Module 1: Méthodes d'orientation, orientation professionnelle interculturelle

Module 2: Diagnostic, évaluation, tests dans l'orientation professionnelle transnationale

Module 3: Méthodologie d'orientation et d'information de divers groupes cibles (orientée vers l'offre - orientée vers la demande)

3. PLACEMENT, COOPÉRATION avec les ENTREPRISES, SUIVI (follow-up)

Module 1: Marché du travail (régional, national, européen)

Module 2: Méthodes de placement

- aptitude

- analyse du poste de travail

- placement: médial - personnel

- analyse d'offres d'emploi

Module 3: Programmes et réseaux nationaux et européens pour le placement (EURES, ACADEMIA-UE, euroguidance)

4. NETWORKING, PROGRAMMATION, MARKETING, CONTRÔLE de GESTION et ÉVALUATION

Module 1: Réseaux nationaux/régionaux

Module 2: Réseaux européens (NRCVG, EURES, etc.)

Module 3: Fédérations intenationales (CEDEFOP, ETF, ILO, Banque mondiale, AIOSP)

Module 4: Marketing: produit, communication, distribution, politique des prix

Module 5: Contrôle de gestion et évaluation

5. ACTIONS DE QUALIFICATION des conseillers, ÉCHANGE, RECHERCHE

Cependant, au sens des études présentées au début, en particulier de l'étude de l'OCDE, pour les conseillers il faudrait exiger un profil de professionnalisme unique. Ce qui fait partie de ce professionnalisme est

a) une qualification spécialisée et systématique basée sur les sciences,

b) l'obligation d'observer un code de déontologie (standards éthiques) et

c) l'affiliation à une organisation de métier qui contrôle constamment les compétences et l'observation des normes.

Pour remplir la condition a), il faut sans doute des études universitaires, où l'on peut distinguer, toutefois, deux directions d'après une comparaison internationale: une orienta- 


\section{TABLEAU 3: Types de formation des conseillers en comparaison internationale}

1. Études spécialisées (3 années) à l'Université ou Ecoles supérieures spéciales avec un grade universitaire au niveau Bachelor of Arts (B.A.), Diplom (FH)

\section{D, NL, S, CH, (A), H, PL}

2. Études postgraduées (1 - 2 années) avec le grade Master of Arts (M.A.), Diplom (Univ.)

\section{USA, Canada, CH (Lizentiat), (F), P, H, PL, Estonia, Bulgaria}

3. Cours internes (Programme formalisé)

\section{DK, F, D, GR, IRL, UK}

4. Spécialisation au sein d'un cycle d'études général: Psychologie, Pédagogie, Sociologie

$$
\text { F, H, PL, B, L, P, E }
$$

5. Cours non-formalisés (interne et externe)

DK, L, UK, GR, IRL, NL

tion explicite vers la psychologie, ce qu'on trouve p.ex. en France, en la Suisse et en partie en Pologne (Université de Lodz). D'autres nombreux pays ont des cours d'études multidisciplinaires soit à dominante en sciences sociales (p.ex. U.S.A., Canada) soit ayant, en dehors de sciences sociales (psychologie, pédagogie, sociologie), une grande partie de sciences économiques et de jurisprudence (p.ex. Allemagne).

Indépendamment de la question de la structure du contenu, quelques indices laissent penser que la tendance internationale dans la qualification des conseillers va vers des études post-diplôme (1-2 années, en cas de formation parallèle avec une prolongation adaptée) avec un diplôme «master»; dans certains pays, en gardant les existantes formes d'études.

Pour la condition b) on peut invoquer les standards éthiques de l'Association internationale (AIOSP) de 1996, qui s'appuient sur les standards nord-américains généralement reconnus. Toutefois, il faudrait harmoniser le règlement juridique-institutionnel existant au niveau national pour l'orientation professionnelle avec ce code de déontologie, chose qui sans doute ne pose aucun problème en ce qui concerne les «principes» de la Bundesanstalt für Arbeit (BA).

La condition c) dépend de l'existence d'organisations professionnelles reconnues dans les pays européens. Il y a des différences considérables, surtout en ce qui concerne la possibilité de contrôle et de sanction, le cas échéant, par une organisation de métier, des grands prestataires d'orientation professionnelle, comme la BA p. ex. Quant à l'Allemagne, il semble plus réaliste de confier à la BA l'admission, l'obligation d'observer un code de déontologie, et le contrôle des conseillers, en étroite collaboration avec l'Association allemande d'orientation professionnelle $(\mathrm{dvb})$.

\section{Références}

Commission of the European Communities (2000). A Memorandum on Lifelong Learning. Brussels (Commission Staff Working Paper).

Ertelt, B. J. \& Schulz, W. E. (1997). Beratung in Bildung und Beruf. Leonberg (Rosenberger). 
OECD/OCDE (Organisation for Economic Co-operation and Development) (1996): Mapping the Future - Young People and Career Guidance. Paris: Cedex.

Public Empoloyment Services (PES/S.P.E.) (2000). «Avenir des Services Publics de l'Emploi (S.P.E.)», Déclaration de mission coummune des S.P.E. en Europe (U.E./E.E.E.).

WATTS, A. G., in association with J. Guichard, P. Plant, M. L. Rodriguez (1993). Education and vocational guidance in the European Community - Synthesis Report. Brussels: EU-Commission.

Fecha de recepción: 10-IX-01

Fecha de revisión: 25-IX-01

Fecha de aceptación: 16-X-01 